\title{
Deterministic constant-temperature dynamics for dissipative quantum systems
}

\author{
Alessandro Sergi* \\ Dipartimento di Fisica, Universitá degli Studi di Messina, Contrada Papardo 98166 Messina, Italy
}

\begin{abstract}
A novel method is introduced in order to treat the dissipative dynamics of quantum systems interacting with a bath of classical degrees of freedom. The method is based upon an extension of the Nosè-Hoover chain (constant temperature) dynamics to quantum-classical systems. Both adiabatic and nonadiabatic numerical calculations on the relaxation dynamics of the spin-boson model show that the quantum-classical Nosè-Hoover chain dynamics represents the thermal noise of the bath in an accurate and simple way. Numerical comparisons, both with the constant energy calculation and with the quantum-classical Brownian motion treatment of the bath, show that the quantum-classical Nosè-Hoover Chain dynamics can be used to introduce dissipation in the evolution of a quantum subsystem even with just one degree of freedom for the bath. The algorithm can be computationally advantageous in modeling, within computer simulation, the dynamics of a quantum subsystem interacting with complex molecular environments.
\end{abstract}

One of the most natural ways to make a quantum system follow a dissipative dynamics is achieved by putting it into contact with a thermal bath. Since usually one is not interested in the detailed time evolution of the bath degrees of freedom, it may also be convenient to approximate the bath dynamics by means of a classical description. When one faces with the problem of calculating the influence of an environment over a quantum subsystem, this approach leads to the representation of (a certain class of) open quantum systems [1] by means of mixed quantum-classical theories. Examples can be found in many phenomena connected to quantum optics 2] and quantum information theory [3]. Typically, this is the case of cosmology where, due to the perduring lack of a full quantum theory of gravitation, one is forced to approximate formalisms in order to treat the interaction of quantum and classical degrees of freedom [4]. In many situations, condensed-matter quantum systems at finite temperature can also be treated with mixed quantum-classical theories. In light of the above discussion, one can certainly conclude that mixed quantumclassical approximations can be used in open quantum systems to describe many processes which are relevant to various fields of research. It is worth noting that mixed quantum-classical theories [5] applied to condensed-matter systems can treat classical molecular bath which can be as complex as state-of-the-art molecular dynamics simulation techniques permit nowadays.

In the original constant-energy (NVE) formulation, mixed quantum-classical algorithms require many environmental degrees of freedom in order to describe the dissipative dynamics of the quantum subsystem. This has been shown within a path-integral influence functional approach [6]. However, quantum-classical dynamics has been recently generalized [7] in order to be unified with the constant-temperature simulation method originally developed by Nosè and Hoover [8] (more generally, the author in Ref. [7] proposed a scheme in order to unify quantum-classical dynamics with many energy-preserving phase space flows [9]). Therefore, one can think of using quantum-classical Nosè-Hoover $(\mathrm{NH})$ dynamics in order to describe dissipative effects and, in particular, the constant-temperature relaxation dynamics of a relevant quantum subsystem. In practice, in order to overcome possible problems with ergodicity in classical phase space, it is more convenient to generalize the Nosè-Hoover chain (NHC) method of Martyna and coworkers 10] to the quantum-classical case and to adopt it in place of the NH dynamics. However, the choice of the NHC dynamics can be viewed as a mere technical point with no deep conceptual implication as far as quantum-classical theories are concerned. In this Communication, by simulating the relaxation dynamics of the spin-boson model [11], I show that the quantum-classical NHC dynamics can be adopted in order to describe dissipative effects in quantum-classical systems by means of a minimal (with respect to the number of degrees of freedom explicitly taken into account) representation of the classical bath.

The quantum-classical Hamiltonian of the spin-boson model reads

$$
\hat{H}_{\mathrm{sb}}=-\hbar \Omega \hat{\sigma}_{x}+\sum_{j=1}^{N_{b}}\left(\frac{P_{j}^{2}}{2 M_{J}}+\frac{1}{2} M_{j} \omega_{j}^{2} R_{j}^{2}-c_{j} R_{j} \hat{\sigma}_{z}\right)
$$

where $2 \hbar \Omega$ is the energy gap of the isolated two-state system, $\hat{\sigma}_{z}$ and $\hat{\sigma}_{x}$ are Pauli's matrices, $R_{j}$ and $P_{j}$ are the coordinates and momenta, respectively, of $N_{b}$ harmonic oscillators with mass $M_{j}$ and frequencies $\omega_{j}$ making up the classical bath. The other parameters of the system, i.e., $\left(M_{j}, \omega_{j}, c_{j}\right)$, can be fixed by requiring that the harmonic bath is described by an Ohmic spectral density. In order to study the relaxation dynamics of this model [12], one

\footnotetext{
* E-mail: asergi@unime.it
} 


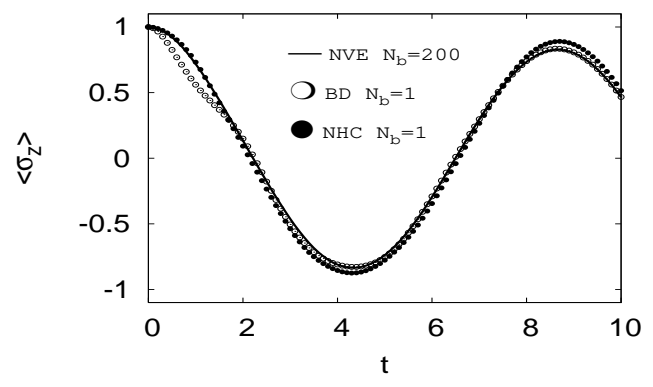

FIG. 1: Adiabatic dynamics of the spin-boson model: $\beta=0.3, \Omega=1 / 3, \omega_{\max }=3, \xi=0.007$. The continuous black line represents the NVE results with $N_{b}=200$; the white circles represent the BD results with $N_{b}=1$ and $\zeta=1$; the black circles represent the NHC results with $N_{b}=1$.

can assume that the system is initially in an uncorrelated state with the quantum subsystem in state $\mid$ up $\rangle$ and the classical harmonic bath in thermal equilibrium. The corresponding quantum-classical density matrix can be found starting from the full quantum expression by means of a partial Wigner transform [13] and was explicitly written down in Ref. [12]. The NVE quantum-classical dynamics of this system is formally exact [12] (i.e., the quantum-classical equations of motion have the same form that would arise within a full quantum treatment) and numerical results, which agree very well with those obtained by means of more sophisticated path-integral iterative techniques [6], are available in the literature [12]. Such NVE results, which were obtained with $N_{b}=200$, will be compared here with those obtained with calculations performed by means of the quantum-classical NHC dynamics and with the bath made up by just one harmonic oscillator $\left(N_{b}=1\right)$.

For the spin-boson model, the quantum-classical NHC dynamics can be defined upon introducing an extended Hamiltonian with a chain of just two thermostat variables

$$
\hat{H}_{(\mathrm{NHC})}=\hat{H}_{\mathrm{sb}}+\frac{p_{\eta_{1}}^{2}}{2 m_{\eta_{1}}}+\frac{p_{\eta_{2}}^{2}}{2 m_{\eta_{2}}}+N_{b} k_{B} T \eta_{1}+k_{B} T \eta_{2},
$$

where $T$ is the temperature of the bath thermalizing the quantum spin, $\eta_{1}, \eta_{2}, p_{\eta_{1}}, p_{\eta_{2}}$ are the Nosè variables, and $m_{\eta_{1}}, m_{\eta_{2}}$ are fictitious masses. Following Ref. [7], the quantum-classical NHC bracket can be defined as:

$$
\begin{aligned}
\left(\begin{array}{ll}
\hat{H}_{(\mathrm{NHC})} & \left., \hat{\sigma}_{z}\right)_{(\mathrm{NHC})}=\frac{i}{\hbar}\left[\hat{H}_{(\mathrm{NHC})}\right. \\
& \cdot\left[\begin{array}{cc}
0 & \hat{\sigma}_{z}
\end{array}\right] \\
-1-\frac{\hbar \Lambda^{(\mathrm{NHC})}}{2 i} & 1+\frac{\hbar \Lambda^{(\mathrm{NHC})}}{2 i} \\
-1 & 0
\end{array}\right] \cdot\left[\begin{array}{c}
\hat{H}_{(\mathrm{NHC})} \\
\hat{\sigma}_{z}
\end{array}\right]
\end{aligned}
$$

where $\Lambda^{(\mathrm{NHC})}$ is a bracket operator whose action between two quantum-classical variables is defined as:

$$
\hat{\xi}(X) \Lambda^{(\mathrm{NHC})} \hat{\chi}(X)=-\sum_{I J} \frac{\partial \hat{\xi}}{\partial X_{I}} \mathcal{B}_{I J}^{(\mathrm{NHC})} \frac{\partial \hat{\chi}}{\partial X_{J}} .
$$

Adopting as a convention for the point of the extended Nosè phase space $X=\left(R, \eta_{1}, \eta_{2}, P, p_{\eta_{1}}, p_{\eta_{2}}\right)$, the antisymmetric NHC matrix reads:

$$
\mathcal{B}^{(\mathrm{NHC})}=\left[\begin{array}{cccccc}
0 & 0 & 0 & 1 & 0 & 0 \\
0 & 0 & 0 & 0 & 1 & 0 \\
0 & 0 & 0 & 0 & 0 & 1 \\
-1 & 0 & 0 & 0 & -P & 0 \\
0 & -1 & 0 & P & 0 & -p_{\eta_{1}} \\
0 & 0 & -1 & 0 & p_{\eta_{1}} & 0
\end{array}\right] .
$$

It is worth noting that, since the Nosè coordinates are intrinsically classical, a quantum-classical treatment of such a constant-temperature dynamics is conceptually correct and, moreover, allows one to address nonadiabatic effects. Other approaches [14], which do not use a quantum-classical bracket, do not seem to permit nonadiabatic calculations 


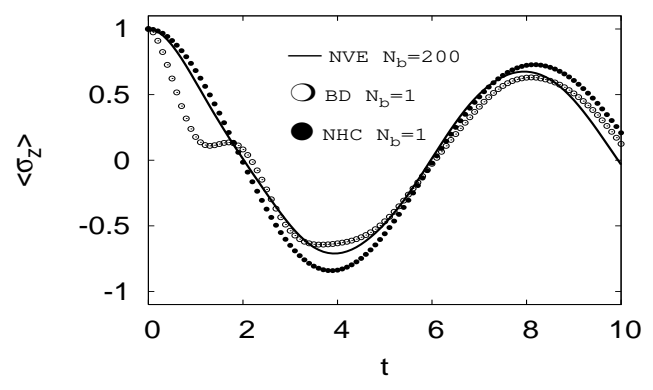

FIG. 2: Adiabatic dynamics of the spin-boson model: $\beta=3, \Omega=1 / 3, \omega_{\max }=3, \xi=0.1$. The continuous black line represents the NVE results with $N_{b}=200$; the white circles represent the BD results with $N_{b}=1$ and $\zeta=1$; the black circles represent the NHC results with $N_{b}=1$.

in a straightforward manner. The equations of motion can be written in the adiabatic basis as

$$
\frac{d}{d t} \chi^{\alpha \alpha^{\prime}}(X, t)=\sum_{\beta \beta^{\prime}} i \mathcal{L}_{\alpha \alpha^{\prime}, \beta \beta^{\prime}}^{(\mathrm{NHC})} \chi^{\beta \beta^{\prime}}(X, t)
$$

where

$$
\begin{aligned}
i \mathcal{L}_{\alpha \alpha^{\prime}, \beta \beta^{\prime}}^{(\mathrm{NHC})} & =i \omega_{\alpha \alpha^{\prime}} \delta_{\alpha \beta} \delta_{\alpha^{\prime} \beta^{\prime}}+i L_{\alpha \alpha^{\prime}}^{(\mathrm{NHC})} \delta_{\alpha \beta} \delta_{\alpha^{\prime} \beta^{\prime}} \\
& -J_{\alpha \alpha^{\prime}, \beta \beta^{\prime}} \\
i L_{\alpha \alpha^{\prime}}^{(\mathrm{NHC})} & =\frac{1}{2} \sum_{I J} \mathcal{B}_{I J}^{(\mathrm{NHC})} \frac{\partial\left(H_{(\mathrm{NHC})}^{\alpha}+H_{(\mathrm{NHC})}^{\alpha^{\prime}}\right)}{\partial X_{J}} \frac{\partial}{\partial X_{I}},
\end{aligned}
$$

and $\omega_{\alpha \alpha^{\prime}}=\left(E_{\alpha}(R)-E_{\alpha^{\prime}}(R)\right) / \hbar$. The quantum transitions operator, $J$, is defined as in the constant-energy case [7]. One wants to calculate the time-dependent quantum-classical average:

$$
\left\langle\hat{\sigma}_{z}(X, t)\right\rangle=\sum_{\alpha \alpha^{\prime}} \int d X \rho_{W}^{\alpha^{\prime} \alpha} \sigma_{z}^{\alpha \alpha^{\prime}}(X, t)
$$

where $\sigma_{z}^{\alpha \alpha^{\prime}}(X, t)$ is given by Eq. (6). Details of the numerical algorithm for calculating Eq. (9) both in the adiabatic and nonadiabatic limit, can be found in Ref. 12]. It is useful to recall that the nonadiabatic quantum-classical dynamics can be pictured as a piece-wise deterministic propagation of the classical phase space point $X$ over the energy surface $\left(\alpha \alpha^{\prime}\right)$ interspersed by stochastic quantum transitions (realized by the action of $J$ ). Note also that, in the NVE case, one must have $N_{b} \geq 200$, as proven by a cumulant expansion analysis of the influence functional entering the path-integral iterative procedure of Ref. [6].

In principle, dissipation might also be described by means of the quantum-classical Brownian dynamics (BD) that was introduced in Ref. [15]. In such a case, the quantum-classical average of $\hat{\sigma}_{z}$ is still given by an equation similar to (9) where, however, the time evolution is achieved by means of a quantum-classical Langevin-Liouville operator, whose explicit expression in the adiabatic basis is known [15]. Such a stochastic operator is defined in terms of a friction constant, $\zeta$, and of a Gaussian white noise process, $\xi(t)$, with the properties $\langle\xi(t)\rangle=0$, and $\left\langle\xi(t) \xi\left(t^{\prime}\right)\right\rangle=2 k_{B} T \zeta \delta\left(t-t^{\prime}\right)$. Therefore, it is interesting to check whether the Brownian dynamics of a bath with $N_{b}=1$ can also lead to an accurate dissipative dynamics. However, as shown in the following, especially when considering nonadiabatic effects, the numerical results prove that the NHC quantum-classical dynamics provides a scheme which is much more accurate and robust than that arising from the Brownian dynamics.

The spin-boson model has been simulated by using dimensionless coordinates [12]. Hundred thousand trajectories were produced in order to sample the initial condition in the nonadiabatic calculations [12]. The system parameters were $\Omega=1 / 3$ and $\omega_{\max }=3$, while the Kondo parameter and the reduced temperature took the two sets of values $(\xi=0.007, \beta=0.3)$ and $(\xi=0.1, \beta=3)$. The results of the NVE calculation, with a bath composed of $N_{b}=200$ oscillators, were compared with those obtained with just one oscillator $\left(N_{b}=1\right)$ in the two cases where either the NHC dynamics or the Brownian dynamics is used. The outcome is that in the NHC dynamics a single oscillator provides a good numerical representation of the dissipative quantum dynamics of the spin. Moreover, it turns out 


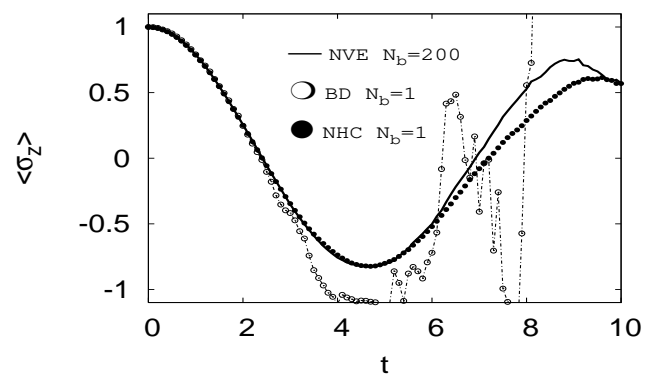

FIG. 3: Non-adiabatic dynamics of the spin-boson model including up to 6 quantum transitions: $\beta=0.3, \Omega=1 / 3, \omega_{\text {max }}=3$, $\xi=0.007$. The continuous black line represents the NVE result with $N_{b}=200$; the black circles represent the NHC results with $N_{b}=1$; the white circles (joined with a dashed line in order to help the eye) represent the BD results with $N_{b}=1$ and $\zeta=1$.

that when nonadiabatic transitions are taken into account, the quantum-classical NHC dynamics provides very good results while the Brownian dynamics fails badly. Note that the the figures in this letter display the results of BD calculations performed only with $\zeta=1$ (in dimensionless units). However, various other calculations were performed, with effective values of $\zeta$ between 0 and 10, without obtaining any improvement in the nonadiabatic case. Figures 1 and 2 show the results of the adiabatic calculations for the set of parameters $(\xi=0.007, \beta=0.3)$ and $(\xi=0.1, \beta=3)$, respectively. In the adiabatic case, both the NHC and Brownian dynamics describe well the dissipative evolution of the quantum subsystem interacting with a single oscillator. The inclusion of nonadiabatic transitions (up to six for each trajectory in the quantum-classical ensemble) shows that, with $N_{b}=1$, the NHC dynamics is still very accurate while the BD evolution becomes numerically unstable at short times. This is not completely unexpected since when the system can switch from one potential surface to another, because of the nonadiabatic transitions, the BD dynamics (in the case of $N_{b}=1$ ) lacks of any equilibrating mechanism. Instead, the quantum-classical NHC dynamics still conserves the Hamiltonian along the trajectory. Such a conservation provides a robust stabilization mechanism even for calculations with baths with very few degrees of freedom. Figure 3 shows the nonadiabatic results for the set of parameters $(\xi=0.007, \beta=0.3)$ while those obtained with the set $(\xi=0.1, \beta=3)$ are displayed in Fig. 4 . In general, the results obtained with the NHC nonadiabatic evolution appear to be numerically more stable and smoother than those obtained with NVE dynamics. This is even more apparent in a slightly stronger coupling regime (see Fig. 4).

It must be remarked that surface-hopping calculations within nonadiabatic quantum-classical dynamics are, for the moment, limited to relatively short times because of numerical instabilities [12]. To address this issue, a quantumclassical non-linear formalism has been recently proposed [16]. However, such long-time integration problems are not related to the NHC dynamics but challenge quantum-classical approximations of quantum dynamics on a more general level. In order to clarify this point, Fig. [5] displays the results of a long-time calculation, performed in the adiabatic approximation. Since there is a great interest in the phenomenon of driven quantum tunneling [17], a static perturbation of the form $\hat{H}_{\text {ext }}=-\hbar \gamma_{s} \hat{\sigma}_{z}$ was added to the unperturbed spin-boson Hamiltonian in Eq. (11), and simulations were carried out both in the $\operatorname{NVE}\left(N_{b}=200\right)$ and NHC case $\left(N_{b}=1\right)$ with $\gamma_{s} / \hbar=(1 / 3) \Omega$ while the other system parameters took the same values as in the calculations whose results are illustrated in Figs. 1 and 3 . The results displayed in Fig. 5 s shows clearly that, in the adiabatic approximation, the numerical agreement between the NVE dynamics $\left(N_{b}=200\right)$ and the NHC dynamics $\left(N_{b}=1\right)$ is very good even over long time intervals.

The results provided in this Communication suggest the possibility of representing the environmental noise, leading to dissipative quantum dynamics, by means of deterministic NHC quantum-classical dynamics. Such an idea can have deep conceptual implications since there is a subtle connection between thermal and quantum fluctuations [18]. Moreover, the algorithms presented here might open novel advantageous routes for the computer simulation of quantum dynamics in open molecular environments. Within condensed matter systems, an example that might be studied by means of the approach illustrated in this Communication is provided by the system recently investigated in Ref. [19]: A retinal chromophore molecule evolving according to short-time quantum coherent dynamics in bacteriorhodopsin. As already shown in Ref. [20], a minimal model of such chromophore-protein systems can be built by explicitly considering the chromophore molecule itself and the nearest-neighbor amino-acids, belonging to the tight-binding pocket in which the chromophore is contained. On the short-time scale of the coherent quantum dynamics of the chromophore, one might think of representing the dissipation entailed by the rest of the protein by means of a deterministic NHC dynamics with a minimal bath. It is also worth to note that there is a great interest in the field of quantum information in the phenomenon of driven quantum tunneling [17]. In particular, recent work focuses on time-dependent external driving [21]. In order to deal with such situations, one should generalize the quantum-classical approach presented 


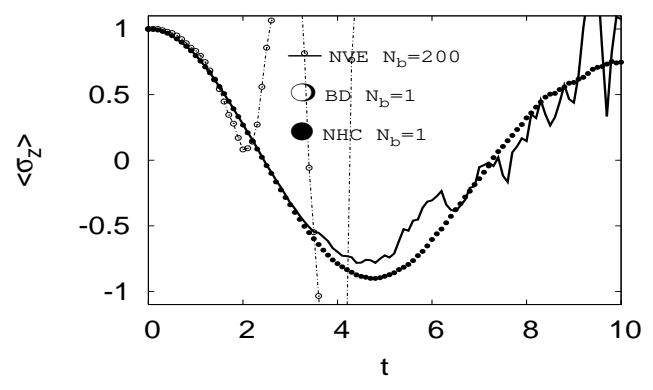

FIG. 4: Nonadiabatic dynamics of the spin-boson model including up to 6 quantum transitions: $\beta=3, \Omega=1 / 3, \omega_{\text {max }}=3$, $\xi=0.1$. The continuous black line represents the NVE results with $N_{b}=200$; the black circles represent the NHC results with $N_{b}=1$; the white circles (joined with a dashed line in order to help the eye) represents the BD with $N_{b}=1$ and $\zeta=1$.

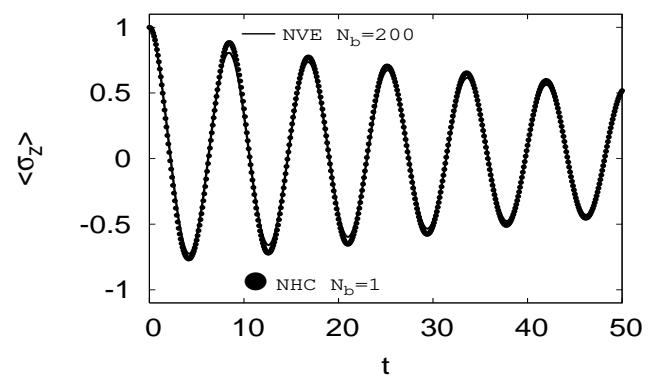

FIG. 5: Adiabatic dynamics of the spin-boson model under the action of a static perturbation of the form $\hat{H}_{\text {ext }}=-\hbar \gamma_{s} \hat{\sigma}_{z}$ : $\beta=3, \Omega=1 / 3, \omega_{\max }=3, \xi=0.1, \gamma_{s} / \hbar=(1 / 3) \Omega$. The continuous black line represents the NVE results with $N_{b}=200 ;$ the black circles represent the NHC results with $N_{b}=1$.

here in order to unify it with the techniques of non-equilibrium molecular dynamics simulations. Although non-trivial algorithmic issues might be expected, this is something which is possible in principle and that deserves a thorough future investigation.

In conclusion, the quantum-classical NHC dynamics may find interesting applications in various fields: In fact, it may be used to simulate not only systems in the fields of chemical-physics or biophysics but also in quantum-optics [2] and quantum computing [3].

\section{Acknowledgments}

I am grateful to Professor Paolo V. Giaquinta and Dr. Giuseppe Pellicane for a critical reading of the manuscript.

[1] H.-P. Breuer and F. Petruccione, The theory of open quantum systems (Oxford University Press, Oxford, 2003).

[2] H. Paul, Introduction to Quantum Optics: From Light Quanta to Quantum Teleportation (Cambridge University Press, Cambridge, 2004); L. Mandel and E. Wolf, Optical Coherence and Quantum Optics (Cambridge University Press, Cambridge, 1995).

[3] M. Le Bellac, A Short Introduction to Quantum Information and Quantum Computation (Cambridge University Press, Cambridge, 2006).

[4] R. M. Wald, Quantum Field Theory in Curved Spacetime and Black Hole Thermodynamics (The University of Chicago, Chicago, 1994); N. D. Birrell and P. C. W. Davies, Quantum fields in curved space (Cambridge University Press, Cambridge, 1994).

[5] I. V. Aleksandrov, Z. Naturforsch., 36a, 902 (1981); V. I. Gerasimenko, Theor. Math. Phys., 50, 77 (1982); D. Ya. Petrina, V. I. Gerasimenko and V. Z. Enolskii, Sov. Phys. Dokl., 35, 925 (1990); W. Boucher and J. Traschen, Phys. Rev. D, 37, 3522 (1988); W. Y. Zhang and R. Balescu, J. Plasma Phys., 40, 199 (1988); R. Balescu and W. Y. Zhang, J. Plasma Phys. 40, 215 (1988); O. V. Prezhdo and V.V. Kisil, Phys. Rev. A, 56, 162 (1997); C. C. Martens and J.-Y. Fang, J. Chem. Phys. 106, 4918 (1996); A. Donoso and C. C. Martens, J. Phys. Chem. 102, 4291 (1998); R. Kapral and G. Ciccotti, J. Chem. Phys., 110, 8919 (1999).

[6] N. Makri and K. Thompson, Chem. Phys. Lett. 291, 101 (1998); K. Thompson and N. Makri, J. Chem. Phys. 110, 1343 
(1999); N. Makri, J. Phys. Chem. B 103, 2823 (1999).

[7] A. Sergi, Phys. Rev. E 72066125 (2005).

[8] S. Nosè, Mol. Phys. 52, 255 (1984); W. G. Hoover, Phys. Rev. A 31, 1695 (1985); S. Nosè, Prog. Theor. Phys. 103, 1 (1991).

[9] A. Sergi, J. Chem. Phys. 124, 024110 (2006); Atti Accad. Pelorit. Pericol. Cl. Sci. Fis. Mat. Nat. 33 c1a0501003 (2005); Phys. Rev. E 72031104 (2005); Phys. Rev. E 69021109 (2004); Phys. Rev. E 67021101 (2003); A. Sergi and M. Ferrario, Phys. Rev. E 64056125 (2001).

[10] G. J. Martyna, M. L. Klein, and M. Tuckerman, J. Chem. Phys. 92, 2635 (1992).

[11] A. J. Leggett, S. Chakravarty, A. T. Dorsey, M. P. A. Fisher, A. Garg, and W. Zwerger, Rev. Mod. Phys. 59, 1 (1987).

[12] A. Sergi, D. Mac Kernan, G. Ciccotti, and R. Kapral, Theor. Chem. Acc. 11049 (2003); D. Mac Kernan, G. Ciccotti, and R. Kapral, J. Chem. Phys. 1162346 (2002).

[13] E. P. Wigner, Phys. Rev. A 40749 (1932); K. Imre, E. Özimir, M. Rosenbaum, and P. Z. Zwiefel, J. Math. Phys. 51097 (1967); M. Hillery, R. F. O'Connell, M. O. Scully, and E. P. Wigner, Phys. Rep. 106121 (1984).

[14] M. Grilli and E. Tosatti, Phys. Rev. Lett. 62, 2889 (1989).

[15] A. Sergi and R. Kapral, J. Chem. Phys. 119, 12776 (2003).

[16] A. Sergi, J. Chem. Phys. 126, 074109 (2007).

[17] M. Grifoni and P. Hänggi, Phys. Rep. 304, 229 (1998).

[18] J. Zinn-Justin, Quantum Field Theory and Critical Phenomena (Oxford University Press, Oxford, 1993); M. Le Bellac, Quantum and Statistical Field Theory (Clarendon Press, Oxford, 1991).

[19] V. Prokhorenko, A. M. Nagy, S. A. Waschuk, L. S. Brown, R. R. Birge, and R. J. Dwayne, Science 313, 1257 (2006).

[20] A. Sergi, M. Grüning, M. Ferrario and F. Buda, J. Phys. Chem. 105, 4386 (2001).

[21] M. C. Goorden, M. Thorwart, and M. Grifoni, Phys. Rev. Lett. 93, 267005 (2004); Eur. Phys. J. B 45, 405 (2005). 